\title{
Institutional Biosafety Committee
}

National Cancer Institute

\section{Source}

National Cancer Institute. Institutional Biosafety Committee. NCI Thesaurus. Code C87779.

An institutional-level board mandated by federal law for oversight of recombinant DNA research and work involving biohazardous agents taking place at that institution. They are charged with assessing the risk of research to the environment and to public health. The board must have at least five members, with at least one with recombinant DNA expertise; at least two members must be unaffiliated with the institution. 